\title{
Restless quiescence: thermonuclear flashes between transient X-ray outbursts
}

\author{
E. Kuulkers ${ }^{1}$, J. J. M. in 't Zand², and J.-P. Lasota ${ }^{3,4}$ \\ 1 ESA, European Space Astronomy Centre (ESAC), PO Box 78, 28691 Villanueva de la Cañada (Madrid), Spain \\ e-mail: Erik.Kuulkers@esa.int \\ 2 SRON Netherlands Institute for Space Research, Sorbonnelaan 2, 3584 CA Utrecht, The Netherlands \\ 3 Institut d'Astrophysique de Paris, UMR 7095 CNRS, UPMC Univ Paris 06, 98bis Bd Arago, 75014 Paris, France \\ 4 Astronomical Observatory, Jagiellonian University, ul. Orla 171, 30-244 Kraków, Poland
}

Received 18 September 2008 / Accepted 11 May 2009

ABSTRACT

\begin{abstract}
For thermonuclear flashes to occur on neutron-star surfaces, fuel must have been accreted from a donor star. However, sometimes flashes are seen from transient binary systems when they are thought to be in their quiescent phase, during which no accretion, or relatively little, is expected to occur. We investigate the accretion luminosity during several such flashes, including the first-ever and brightest detected flash from Cen X-4 in 1969. We infer from observations and theory that immediately prior to these flashes the accretion rate must have been between about 0.001 and 0.01 times the equivalent of the Eddington limit, which is roughly 2 orders of magnitude less than the peak accretion rates seen in these transients during an X-ray outburst and 3-4 orders of magnitude more than the lowest measured values in quiescence. Furthermore, three such flashes, including the one from Cen X-4, occurred within 2 to 7 days followed by an X-ray outburst. A long-term episode of enhanced, but low-level, accretion is predicted near the end of the quiescent phase by the disk-instability model, and may thus have provided the right conditions for these flashes to occur. We discuss the possibility of whether these flashes acted as triggers of the outbursts, signifying a dramatic increase in the accretion rate. Although it is difficult to rule out, we find it unlikely that the irradiance by these flashes is sufficient to change the state of the accretion disk in such a dramatic way.
\end{abstract}

Key words. accretion, accretion disks - binaries: close - binaries: general - stars: neutron - X-rays: binaries - X-rays: bursts

\section{Introduction}

In low-mass X-ray binaries (LMXBs) a neutron or black hole accretes matter via an accretion disk from a less massive Rochelobe filling companion star. In many LMXBs the accretion from the disk on the compact object is transient. After a transient outburst (hereafter referred to as outburst), lasting typically a few months, the system settles in quiescence for a few months to several decades. The disk-instability model (DIM; Osaki 1974, see Lasota 2001, for a review) predicts that, if in quiescence the disk extends down to the stellar surface (or the last stable Keplerian orbit), the accretion rate is negligibly low $\left(\sim 10^{5} \mathrm{~g} \mathrm{~s}^{-1}\right.$; Lasota et al. 2008, see Eq. (1) in Sect. 4). However, in general the X-ray emission of quiescent transient sources corresponds to accretion rates that cannot be qualified as negligible (e.g., van Paradijs et al. 1987; Campana et al. 2004).

In the case of neutron-star transients there has been a controversy on the origin of the quiescent X-ray luminosity. On the one hand, quiescent-disk truncation easily explains the luminosity (Lasota et al. 1996; Dubus et al. 2001; Narayan \& McClintock 2008), is also applicable to black-hole systems, and has been confirmed by observations (e.g., Done 2002). On the other hand, Brown et al. (1998) propose that quiescent X-rays have their origin in heating of the neutron-star crust by nuclear reactions and not in accretion. Both models have their difficulties. The observed rapid variability of the quiescent X-ray flux and the presence of a substantial power-law component (as seen in, e.g., the LMXB transient Cen X-4, see Campana et al. 1997, 2004, Rutledge et al. 2001), as well as a very low quiescent
$\mathrm{X}$-ray luminosity (less than about several times $10^{30} \mathrm{erg} \mathrm{s}^{-1}$ for 1H 1905+000; Jonker et al. 2007), are difficult to reconcile with the deep crustal heating model (see, e.g., Jonker 2008 for a recent discussion). The lack of a well understood disk-truncation mechanism (see, however, Liu et al. 2002) and the overpredicted ratio of neutron-star to black-hole quiescent X-ray luminosities (Menou et al. 1999) are the weaknesses of its competitor. Therefore, any independent estimate of the quiescent accretion rate in transient systems would be a great help in resolving the controversy.

Type I X-ray bursts (Grindlay et al. 1975; Belian et al. 1976; Hoffman et al. 1978) are thermonuclear flashes at the surface of a neutron star (Joss 1977; Maraschi \& Cavaliere 1977; Lamb \& Lamb 1978; for reviews see, e.g., Lewin et al. 1993; Strohmayer \& Bildsten 2006). We hereafter refer to these events as flashes. If the energy release during a flash is fast and large enough, the local luminosity on the neutron star surface can surpass the Eddington limit, resulting in a lift-up of the photosphere. Such flashes are referred to as photospheric radius-expansion X-ray bursts. During the expansion phase the inferred temperature decreases, whereas the inferred emitted area increases (see, e.g., Lewin et al. 1993, for a review).

The first observed flash, in retrospect, was the event detected on July 7, 1969, with Vela 5B from Cen X-4 (Belian et al. 1972; see Sect. 2). The event lasted for about $10 \mathrm{~min}$, and is still the brightest ever observed with a peak flux of about $60 \mathrm{Crab}^{1}$

\footnotetext{
1 The commonly used Crab unit is equivalent to about $2 \times$ $10^{-8} \mathrm{erg} \mathrm{cm}^{-2} \mathrm{~s}^{-1}$ in the classical $2-10 \mathrm{keV}$ photon-energy band.
} 
(3-12 keV). Two days after the flash Cen X-4 went into an X-ray outburst, which peaked at about $25 \mathrm{Crab}(3-12 \mathrm{keV})$ and lasted for about 80 days (Conner et al. 1969; Evans et al. 1970; see also Sect. 2). Except for the flash, no other X-ray emission was detected from Cen X-4 before the outburst (Belian et al. 1972). Interestingly, a similar situation recently occurred in another source: $\mathrm{a} \simeq 40$ s long flash was detected from IGR J17473-2721 about 2 days prior to the X-ray outburst (Del Monte et al. 2008; Markwardt et al. 2008). In an other case three flashes separated by 2-3 days were seen during the beginning of an X-ray outburst of 2S 1803-245 (Cornelisse et al. 2007). Our inspection of the RXTE All Sky Monitor (ASM) light curve shows that the first flash, which lasted for about 40-50 s, occurred when there was no detectable X-ray emission. The following two flashes occurred when the source showed X-ray emission at a slightly elevated level. About a week later the source developed into a bright X-ray outburst.

A few other LMXB transients have shown flashes after and/or in between X-ray outbursts when their X-ray emission was below the detection thresholds and the systems were inferred to be in their quiescent phase: 2S 1711-339, SAX J1808.4-3658 and GRS 1747-312 (Cornelisse et al. 2002a, in 't Zand et al. 2001, 2003b, respectively). Also Cen X-4 may have shown a flash $\sim 2$ years after its 1969 X-ray outburst (Gorenstein et al. 1974; see Appendix A). Related examples are various flashes seen from sources without detectable pre-flash emission, the so-called burst-only sources (Cocchi et al. 2001; Cornelisse et al. 2002a,b, and references therein).

For all the afore-mentioned flashes, accretion must have been ongoing prior to these events at a level that is orders of magnitude lower than that achieved during an outburst, but, interestingly, higher than quiescent levels. This brings about prospects for new constraints on models for the emission during the quiescent phase. We explore in Sect. 3 the observed and expected mass-accretion rates around the times of the flashes seen for the sources presented above. Furthermore, the flashes which are within 2-7 days followed by X-ray outbursts suggest the presence of a physical process which has thus far not been discussed in the literature ${ }^{2}$ : that the flashes serve as triggers for accretiondisk instabilities resulting in X-ray outbursts. We study in Sect. 4 the viability of this idea in the context of Cen X-4.

\section{Cen X-4: revisiting old data}

Cen X-4's 1969 flash was reported to reach a peak flux ${ }^{3}$ of about $1.4 \times 10^{-6} \mathrm{erg} \mathrm{cm}^{-2} \mathrm{~s}^{-1}$. The event rose to an observed maximum

\footnotetext{
${ }^{2}$ Belian et al. (1972) called Cen X-4's 1969 flash a probable precursor to its subsequent $\mathrm{X}$-ray outburst. They suggested the two events to be associated, but no physical scenario was discussed.

3 Whether the flux is bolometric or in the $3-12 \mathrm{keV}$ band is not entirely clear from Belian et al. (1972). Assuming the X-ray detectors onboard Vela $5 B$ to be ideal detectors (i.e., with a $100 \%$ quantum efficiency), we can translate the quoted $3-12 \mathrm{keV}$ count rate $\left(2850 \mathrm{c} \mathrm{s}^{-1}\right.$, Belian et al. 1972) into a photon flux, and subsequently estimate the bolometric flux assuming a black body with a temperature of $3 \times 10^{7} \mathrm{~K}$ (see Belian et al. 1972). This results in a bolometric flux estimate of about $1.8 \times$ $10^{-6} \mathrm{erg} \mathrm{cm}^{-2} \mathrm{~s}^{-1}$, i.e., higher than the quoted peak flux. Since the X-ray detectors are not ideal, quantum efficiencies are lower, which results in even higher estimated bolometric flux values. This and the fact that Belian et al. (1972) speak of an "energy" flux, suggests the quoted peak value to be the $3-12 \mathrm{keV}$ flux. However, given the uncertainties involved and that the quoted peak flux is closer to the bolometric peak flux of that observed during Cen X-4's flash seen with Hakucho, we use the quoted value as the bolometric flux.
}

within up to about $1 \mathrm{~min}$ and lasted about $10 \mathrm{~min}$; the shape of the decay after the peak was more consistent with a power-law than with an exponential (see also below). During the decay the emission softened: at the peak it could be described by a blackbody with a temperature of about $2.6 \mathrm{keV}$, whereas in the tail the temperature was about $1.3 \mathrm{keV}$ (Belian et al. 1972). We note that the effective black-body radius at the peak would be about $6.5 \mathrm{~km}$ (at $1.2 \mathrm{kpc}$, see below). The energy release during the flash was estimated to be about $5 \times 10^{39} \mathrm{erg}$ (at $1.2 \mathrm{kpc}$; Matsuoka et al. 1980). It was recognized later that these are the characteristics of a flash, although it was noted that the event lasted unusually long (Fabbiano \& Branduardi 1979; Matsuoka et al. 1980; Kaluzienski et al. 1980; see also Hanawa \& Fujimoto 1986). However, such long flashes, although rare, have since been seen in other LMXBs as well (see, e.g., Kuulkers 2004; in 't Zand et al. 2007; Falanga et al. 2008).

The position of Cen X-4 has been refined (Hjellming 1979; Canizares et al. 1980) since the early Vela $5 B$ reports; we, therefore, decided to re-analyse the Vela $5 B$ data. The raw data files are archived at HEASARC (see Whitlock et al. 1992; Whitlock \& Tyler 1994). Vela 5B (see Conner et al. 1969) provided the first $\mathrm{X}$-ray all-sky monitor; it was, however, designed to be a nucleartest detection satellite. The satellite rotated about its nadir-fixed spin axis with a 64-s period, and orbited the Earth in around $112 \mathrm{~h}$. The scintillation X-ray detector (XC) was located at about $90^{\circ}$ from the spin axis, and so covered the X-ray sky twice per satellite orbit. Data were telemetered in 1-s count accumulations. The X-ray detector provided data in two energy channels, 3$12 \mathrm{keV}$ and $6-12 \mathrm{keV}$. A slat collimator limited the field of view to a $F W H M$ aperture of $6.1^{\circ} \times 6.1^{\circ}$; the effective detector area was about $26 \mathrm{~cm}^{2}$.

We extracted the light curves, assuming that no other strong $\mathrm{X}$-ray sources were in the field of view. We used two time scales. For the X-ray outburst we used 56 h (i.e., half the Vela $5 B$ satellite orbital period), and included data up to the recommended $5^{\circ}$ off-axis from the position of Cen X-4. For the flash we used the 1 -s info. The beginning of the flash occurred at a larger off-axis angle than the recommended value; here we therefore included data from up to $6^{\circ}$. Data flagged for an unstable spin period and for pointing errors were not included. The data were corrected using a sinusoidally modelled background. For some sources, removal of the fitted background leaves a slightly negative average in the count rate; this is also the case for Cen X-4. We corrected the 56-h average light curve for the observed negative average in the count rate just before and after the X-ray outburst (about $-3 \mathrm{c} \mathrm{s}^{-1}$ ). The 1-s data are corrected for the collimator response and the time stamps are corrected to solar-system barycentric time. The errors on these data are determined as follows: we assumed Poisson counting statistics in the light curves uncorrected for background and collimator; the errors were then propagated when all corrections were made. The 56-h count rates are weighted averages of the 1-s data; the errors take into account uncertainties introduced by background removal and collimator response correction, as well as counting statistics (see Whitlock et al. 1992). We normalized the count rates to the Vela 5B Crab rate observed in the $3-12 \mathrm{keV}$ band between 1969, May 28 and Oct. 29, i.e., around the time of the 1969 X-ray outburst $\left(43.2 \pm 0.2 \mathrm{c} \mathrm{s}^{-1}\right)$.

The flash (Fig. 1, left panel) rose to the observed peak within up to $62 \mathrm{~s}$. We find a peak flux of $57 \pm 2 \mathrm{Crab}$, which is more than about 3 orders of magnitude above the upper limits on the pre-flash level (see Fig. 1, right panel). Our revised peak intensity is a factor of $\simeq 1.15$ lower than that reported by Belian et al. (1972). Assuming the count rate and black-body flux scale 

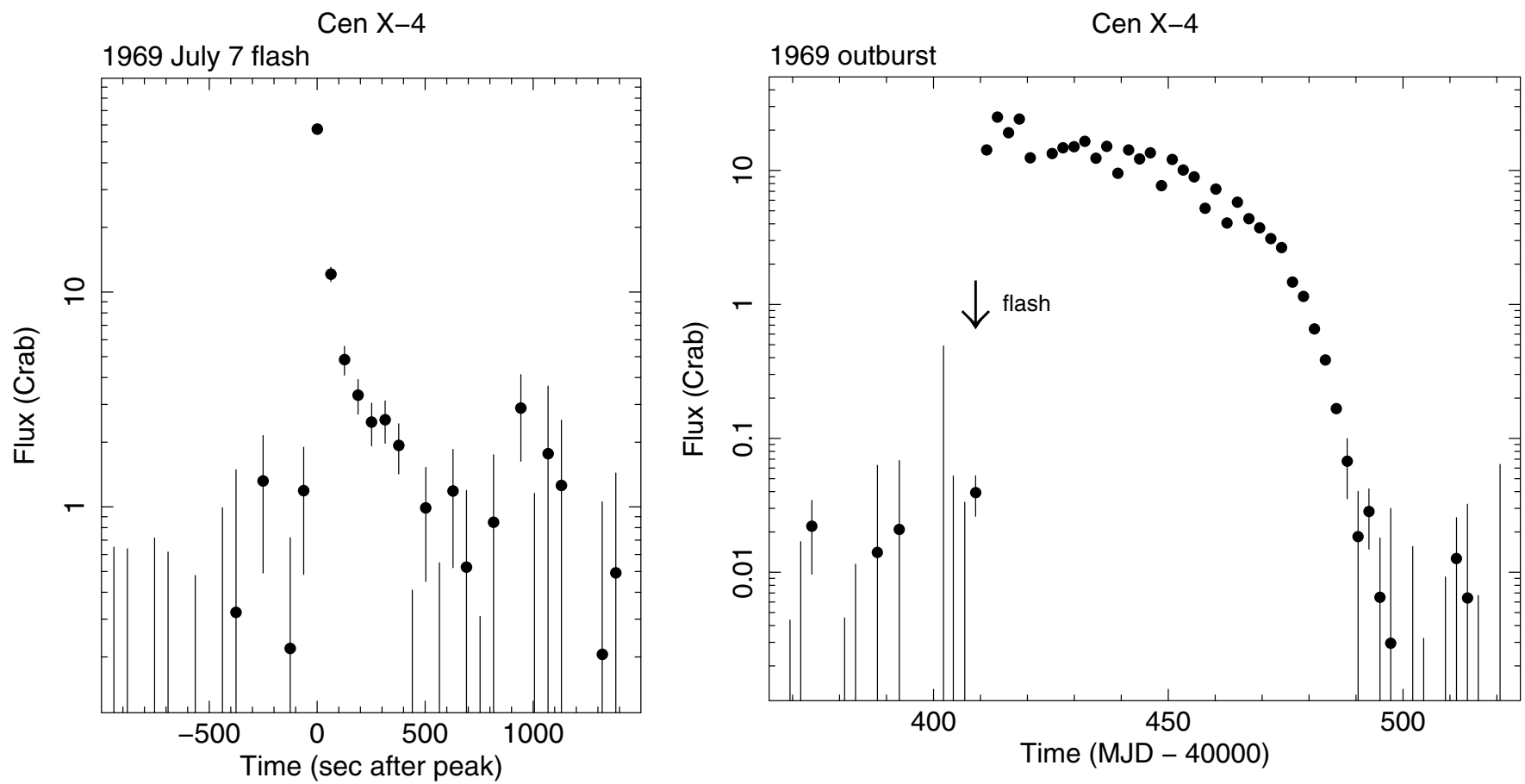

Fig. 1. Left: Vela $5 B$ light curve (3-12 keV) of the X-ray event from Cen X-4 which occurred on July 7, 1969. Time $=0 \mathrm{~s}$ corresponds to UT 01:56:56. See also Belian et al. (1972). Right: Vela 5B light curve (3-12 keV) of the X-ray outburst of Cen X-4 which lasted from 1969, July-September. See also Evans et al. (1970). Data are shown from near the start (1969 May 28) up to about 155 days after the start (1969 Oct. 29 ) of the Vela 5B mission. The time of the flash is indicated with an arrow.

linearly, we infer a peak flux of about $1.2 \times 10^{-6} \mathrm{erg} \mathrm{cm}^{-2} \mathrm{~s}^{-1}$. We only have sparse timing information. Because of the data gaps the actual peak flux may have been even higher; we also can not say with certainty whether the event showed a photospheric radius-expansion phase indicative of (super-)Eddington fluxes from the flash or not.

Until about $150 \mathrm{~s}$ after the peak the exponential decay time is about $44 \mathrm{~s}$. After that excess emission above the expected exponential decay is observed. Such excess emission is similar to that seen in other flashes from, e.g., AqlX-1 (Czerny et al. 1987), X1905+000 (Chevalier \& Ilovaisky 1990) and GX 3+1 (Chenevez et al. 2006). Due to this excess emission the shape of the full decay looks more like a power law (see above).

The X-ray outburst (Fig. 1, right panel) reached a peak of $25.0 \pm 0.3 \mathrm{Crab}$, which is about 3 orders of magnitude above the upper limits on the quiescent level. Note that the light curve shows variations on the 56-h time scale (see also Evans et al. 1970). This is attributed to gain variations due to a $60^{\circ}$ satellite temperature change from one side of the satellite orbit to the other. This has not been (and can not be any more) taken into account in the data reduction, because of the lack of pre-launch tests, and the lack of information regarding the temperature values in our time frame of interest (see Whitlock et al. 1992).

A second X-ray outburst occurred in 1979, which reached a peak flux of about $4 \mathrm{Crab}(3-6 \mathrm{keV})$ and lasted about a month (Kaluzienski et al. 1980). During the late stages of the outburst a flash was observed with Hakucho (Matsuoka et al. 1980). This flash reached a peak flux of about 25 Crab (1.5-12 keV; with an estimated uncertainty of about $5 \%$, Matsuoka, private communication) or a bolometric peak flux of $1 \times 10^{-6} \mathrm{erg} \mathrm{cm}^{-2} \mathrm{~s}^{-1}$, attained a peak black-body temperature of $2.5 \pm 0.8 \mathrm{keV}$, lasted for $\simeq 100 \mathrm{~s}$, and had an energy output of about $1 \times 10^{39} \mathrm{erg}$ (at $1.2 \mathrm{kpc}$; Matsuoka et al. 1980). There is no clear evidence of a radius-expansion phase in this flash either.
The peak fluxes of the 1969 and 1979 flashes have been used to estimate the proximity of the system; the upper limit was about $1.2 \mathrm{kpc}$ (Matsuoka et al. 1980; Kaluzienski et al. 1980; Chevalier et al. 1989). Our revised 1969 flash peak flux and the theoretical value for the Eddington-limited luminosity, $L_{\mathrm{Edd}}$ $\left(\simeq 2 \times 10^{38} \mathrm{erg} \mathrm{s}^{-1}\right)^{4}$, for a $1.4 M_{\odot}$ star which accretes material with solar composition (see Sect. 3.1) translate again to an upper limit of about $1.2 \mathrm{kpc}$ (assuming isotropic radiation). This upper limit is consistent with that derived from other constraints, i.e., between $0.9 \mathrm{kpc}$ and $1.7 \mathrm{kpc}$ (González Hernández et al. 2005a). In this paper we adhere to a distance of $1.2 \mathrm{kpc}$.

No significant X-ray emission was detected before and just after the 1969 flash of Cen X-4 (Belian et al. 1972; see also Conner et al. 1969). The 1-day average $3 \sigma$ detection limit of Vela $5 B$ is about 250 Uhuru Flux Units (see Priedhorsky \& Holt 1987), which corresponds to about $6 \times 10^{-9} \mathrm{erg} \mathrm{cm}^{-2} \mathrm{~s}^{-1}$ $(2-10 \mathrm{keV})^{5}$. Using our light curves with $56 \mathrm{~h}$ time resolution during the $\simeq 40$ days before the X-ray outburst we find that the spread in values of the count rates is $32.4 \mathrm{mCrab}$ (3-12 keV). We, therefore, infer a $56 \mathrm{~h}$-average $3 \sigma$ upper limit of $\simeq 97 \mathrm{mCrab}(3-12 \mathrm{keV})$ on the emission before the X-ray outburst. The error on the average of the count rates over the 40 days is $\simeq 6 \mathrm{mCrab}$, from which we infer a $3 \sigma$ upper limit on the emission over the whole 40 -day time interval of $\simeq 17 \mathrm{mCrab}$ (3-12 keV). Assuming a Crab-like spectrum (see, e.g., Kirsch et al. 2005) the above $3 \sigma$ limits translate to $2-10 \mathrm{keV}$ pre-flash fluxes of about $2 \times 10^{-9} \mathrm{erg} \mathrm{cm}^{-2} \mathrm{~s}^{-1}$ and $3 \times 10^{-10} \mathrm{erg} \mathrm{cm}^{-2} \mathrm{~s}^{-1}$,

\footnotetext{
4 The Eddington luminosity as measured by a distant observer is $L_{\mathrm{Edd}}=(4 \pi c G M / \kappa)\left[1-2 G M /\left(R c^{2}\right)\right]^{1 / 2}$, where $c, G, M, \kappa$ and $R$ are the speed of light, the gravitational constant, the mass of the object, the electron scattering opacity and the radius of the object, respectively (see, e.g., Lewin et al. 1993).

51 Uhuru Flux Unit $\left(\right.$ UFU) $=1$ Uhuru c s${ }^{-1}=2.4 \times 10^{-11} \mathrm{erg} \mathrm{cm}^{-2} \mathrm{~s}^{-1}$ (2-10 keV), assuming a Crab-like spectrum (Forman et al. 1978).
} 
respectively. The non-detection of the source during 1977-1978 in the HEAO-A2 sky survey corresponds to an upper limit of about $1 \times 10^{-11} \mathrm{erg} \mathrm{cm}^{-2} \mathrm{~s}^{-1}(2-10 \mathrm{keV}$; see Kaluzienski et al. 1980), indicating that the source approached quiescent values in between the two X-ray outbursts. The quiescent flux observed long after the second $\mathrm{X}$-ray outburst is about $1-3 \times$ $10^{-12} \mathrm{erg} \mathrm{cm}^{-2} \mathrm{~s}^{-1}(0.5-10 \mathrm{keV}$; see Rutledge et al. 2001). It is, however, variable on long-term time scales $(\sim 40 \%$ in 5 years; Rutledge et al. 2001), as well as on short-term time scales (factor of about 3 in a few days and at a level of $\sim 45 \% \mathrm{rms}$ down to about 100 s; Campana et al. 1997, 2004).

\section{Limits on pre-flash accretion rates}

\subsection{Limits from observations}

What is the level of accretion prior to the flashes, allowing them to occur? During none of the flashes mentioned in the Introduction was emission detected just before and after the flash, so in principle no accurate measurement of the luminosity, and therefore accretion rate, can be made around the time of these flashes. Nevertheless, the observed upper limits may still give us useful constraints on the accretion rate.

The ratio of (the upper limit to) the pre-flash emission and the Eddington-limited flux provides an estimate of the fraction of the Eddington limit at which a source is accreting (see, e.g., Cornelisse et al. 2002a). For ease of comparison between the observations and flash theory, we therefore denote the observed X-ray luminosities in terms of the Eddington luminosity, $L_{\mathrm{Edd}}$, and the accretion rates, $\dot{M}$, in terms of the Eddington accretion rate, $\dot{M}_{\text {Edd }}$. The Eddington accretion rate is defined as the accretion rate at which the corresponding accretion luminosity, $L_{\text {acc }}$, equals $L_{\text {Edd }}{ }^{6}$. The secondary star in Cen X-4 is a latetype K3-K7 star in a $\sim 15 \mathrm{~h}$ orbit with the neutron star (e.g., Casares et al. 2007, and references therein). We can fairly assume that the secondary provides a solar mix of $\mathrm{H}$ and $\mathrm{He}$; the metallicity is only slightly over-solar (González Hernández et al. $2005 b)$. For the other systems this is not clear ${ }^{7}$. Assuming that the metallicity of these sources does not deviate much from solar, $L_{\text {Edd }} \simeq 2 \times 10^{38} \mathrm{erg} \mathrm{s}^{-1}$ (see Sect. 2 ), while correspondingly $\dot{M}_{\text {Edd }} \simeq 10^{18} \mathrm{~g} \mathrm{~s}^{-1}$ (see footnote 6 , using $L_{\mathrm{acc}}=L_{\mathrm{Edd}}$ ).

Since X-ray instruments provide information in a limited energy range only, one has to correct the observed X-ray fluxes to bolometric values. To determine the bolometric correction factor one has to make assumptions on the spectrum outside the instrument's energy window. For the purpose of this paper we employ a bolometric correction factor of about 2 (see in 't Zand et al. 2001, 2003b, 2007), whenever necessary. We also assume that the pre-flash emission as well as the flash emission is isotropic.

For photospheric radius-expansion flashes the peak bolometric black-body flux, $F_{\text {bb,peak }}$, equals the Eddington flux, and, therefore, $L_{\mathrm{x}, \text { bol }} / L_{\text {Edd }}=F_{\text {pers,bol }} / F_{\text {bb,peak }}$, where $L_{\mathrm{x} \text {,bol }}$ and

${ }^{6} L_{\text {acc }}=G M \dot{M} / R \simeq 1.3 \times 10^{36} \dot{M}_{16}\left(M / M_{\odot}\right)(10 \mathrm{~km} / R) \mathrm{erg} \mathrm{s}^{-1}$, where $L_{\text {acc }}$ is the accretion luminosity and where we used $\dot{M}=10^{16} \dot{M}_{16} \mathrm{~g} \mathrm{~s}^{-1}$. Note that this corresponds to an accretion efficiency of $\sim 0.2$. For an accreting neutron star with a mass of $1.4 M_{\odot}$ and a radius of $10 \mathrm{~km}$, $L_{\mathrm{acc}} \simeq .8 \times 10^{36} \dot{M}_{16} \mathrm{erg} \mathrm{s}^{-1}$. See, e.g., Frank et al. (1992)

7 The secondary of SAXJ1808.4-3658 may be a hot $\sim 0.05 M_{\odot}$ brown dwarf (Bildsten \& Chakrabarty 2001), while the secondary in GRS 1747-312, lying in the globular cluster Terzan 6, may be an ordinary subgiant (in 't Zand et al. 2003a), but no further details are known about their composition, as well as for the other sources we consider in this paper.
$F_{\text {pers,bol }}$ refer to the (estimated) bolometric pre-flash source luminosity and flux, respectively. When a flash does not show such an expansion phase, $F_{\text {bb,peak }}$ represents a lower limit to the Eddington flux, and, therefore, $L_{\mathrm{x}, \text { bol }} / L_{\text {Edd }}<F_{\text {pers,bol }} / F_{\text {bb,peak }}$. The flux values and corresponding luminosity information are shown in Table 1 . Note that the values for $L_{\mathrm{x}, \mathrm{bol}} / L_{\mathrm{Edd}}$ are in the same range as the upper limits derived on the emission of the burst-only sources around the flashes (Cocchi et al. 2001; Cornelisse et al. 2002a, and references therein), where $L_{\mathrm{x}, \text { bol }} / L_{\text {Edd }} \lesssim 0.002-0.02$. The bolometric factor can be uncertain by about a factor of 2 (e.g., in 't Zand et al. 2007), and therefore, $F_{\text {pers,bol }}$ and $L_{\text {x,bol }}$ can be up to about a factor of 2 higher. This does, however, not have an effect on our principal conclusions.

Assuming $L_{\text {x,bol }} / L_{\text {Edd }}$ equals $\dot{M} / \dot{M}_{\text {Edd }}$ (keeping in mind the uncertainties introduced by the bolometric corrections, the assumption here of a radiation efficiency of $\sim 0.2$ and isotropic emission), the above measurements suggest that $\dot{M}$ around the time of the flashes in our sample is $\lesssim 10^{-2} \dot{M}_{\text {Edd }}$, i.e., at least about 2 orders of magnitude lower than what is typically observed when the systems are in outburst. We note here that the lowest measured accretion flux for an active burster is about $0.03 L_{\text {Edd }}$ (in 't Zand et al. 2007).

\subsection{Limits from theory}

The characteristics of a flash (such as duration and peak luminosity) depend primarily on $\dot{M}$ and the composition of the accreted material, for a given neutron star mass (e.g., Fujimoto et al. 1981; Fushiki \& Lamb 1987; see also Peng et al. 2007; Cooper \& Narayan 2007, and references therein). In general, He ignites completely in a fraction of a second, while unstable hydrogen burning is prolonged to about $100 \mathrm{~s}$ through slow beta decays in the rp process (e.g., Cumming 2003; Woosley et al. 2004; Heger et al. 2007; Fisker et al. 2008). The duration of a flash is a convolution of the burning process time and the cooling time. For $\dot{M} \lesssim 10^{-2} \dot{M}_{\text {Edd }}$ flashes occur deeper in the neutron star and the duration is mainly determined by the cooling time. At face value, the duration of the 1969 flash from Cen X-4 is consistent with burning of a H-rich layer with e-folding decay times close to values found for the prototypical burster GS 1826-24 (e.g., Heger et al. 2007; Galloway et al. 2008). However, since the accretion rate at the time of Cen X-4's flash was low, the decay rate is likely to be limited also by the relatively large depth of the ignition. Although the available literature on the theoretical description of the flash behaviour at the low accretion rates relevant to our sample (typically $\lesssim 10^{-2} \dot{M}_{\text {Edd }}$ ) is sparse and has only recently been developed in more detail (Peng et al. 2007; Cooper \& Narayan 2007), we can use it to infer additional constraints on the accretion rate around the time of the observed flashes.

Below $\dot{M}$ of a few times $10^{-3} \dot{M}_{\text {Edd }}$, one expects mixed H/He flashes triggered by thermally unstable $\mathrm{H}$ burning. The decay times of such flashes are on the order of tens of seconds, due to the long waiting times involved in $\beta$-decays. During the peak of these kinds of flashes the fluxes are sub-Eddington, but are expected to exceed about $10 \%$ of the Eddington limit. Between a few times $10^{-3} \dot{M}_{\text {Edd }}$ and $10^{-2} \dot{M}_{\text {Edd }}$, unstable $\mathrm{H}$ burning does not trigger unstable He burning and a layer of $\mathrm{He}$ is being built up, which may eventually lead to an energetic (approaching or even reaching the Eddington limit) and long flash. These $\dot{M}$ limits depend, however, on the emergent flux from the neutron star crust and whether sedimentation is important or not. For example, a factor of 10 higher flux from the crust results in $\dot{M}$ limits which are about a factor of 10 lower, while including 
Table 1. Overview of the properties ${ }^{1}$ of the observed flashes discussed in this paper.

\begin{tabular}{|c|c|c|c|c|c|c|c|c|c|c|}
\hline Source & $\begin{array}{l}F_{\text {bb,peak }}\left(10^{-8}\right. \\
\left.\mathrm{erg} \mathrm{cm}^{-2} \mathrm{~s}^{-1}\right)\end{array}$ & & $\begin{array}{c}F_{\text {pers }} \\
\left(10^{-10} \mathrm{erg} \mathrm{cm}^{-2} \mathrm{~s}^{-1}\right)\end{array}$ & & $\begin{array}{l}F_{\text {pers,bol }} / F_{\text {bb,peak }} \\
\quad \equiv L_{\text {x,bol }} / L_{\text {Edd }}\end{array}$ & $\begin{array}{l}L_{\mathrm{x}, \mathrm{bol}}\left(10^{36}\right. \\
\left.\mathrm{erg} \mathrm{s}^{-1}\right)\end{array}$ & $\begin{array}{l}\Delta t_{\text {last }} \\
\text { (days) }\end{array}$ & & $\begin{array}{l}\Delta t_{\text {next }} \\
\text { (days) }\end{array}$ & \\
\hline Cen X-4 & $\sim 120$ & [1] & $<20^{a}(2-10 \mathrm{keV})$ & [1] & $\lesssim 0.0033$ & $\lesssim 0.7$ & $>40$ & {$[1,2]$} & $\simeq 2$ & {$[1,3]$} \\
\hline 2S 1711-339 & $3.0 \pm 1.0^{b}$ & [4] & $<0.7(2-28 \mathrm{keV})$ & [4] & $\lessgtr 0.0047$ & $\lesssim 1$ & $\sim 340$ & [4] & $\simeq 1440$ & [5] \\
\hline GRS $1747-312$ & $\simeq 2.5^{c}$ & [6] & $<0.6(0.1-200 \mathrm{keV})$ & [6] & $\lessgtr 0.0024$ & $\lesssim 0.5$ & $\simeq 26$ & [6] & $\simeq 28$ & [6] \\
\hline IGR J17473-2721 & $\simeq 11^{d}$ & {$[7]$} & $<1.7(2-10 \mathrm{keV})$ & {$[8]$} & $\lesssim 0.0031$ & $\lesssim 0.4$ & $\sim 960$ & {$[9,10]$} & $\simeq 2$ & {$[8,11]$} \\
\hline $2 \mathrm{~S} 1803-245$ & $3.1 \pm 0.7$ & [12] & $<2^{e}(2-28 \mathrm{keV})$ & [4] & $\lessgtr 0.013$ & $\leqslant 3$ & $\sim 7990$ & [13] & $\simeq 7$ & [12] \\
\hline SAX J1808.4-3658 & $25 \pm 2^{c}$ & [14] & $<3(2-28 \mathrm{keV})$ & [14] & $\lesssim 0.0024$ & $\lesssim 0.5$ & $\simeq 17$ & [14] & $\simeq 543$ & [15] \\
\hline
\end{tabular}

${ }^{a}$ Vela $5 B 56 \mathrm{~h}$ upper limit; ${ }^{b}$ highest peak flux among the flash sample in [4]; ${ }^{c}$ photospheric radius-expansion flash; ${ }^{d}$ peak flux observed during another flash reported by [7]; ${ }^{e}$ no limits are quoted in [12]; we, therefore, assume the highest BeppoSAX/WFC flux upper limit from [4].

Note 1. In the first two columns we give the observed flash bolometric peak fluxes $\left(F_{\mathrm{bb} \text {,peak }}\right)$ and the $3 \sigma$ upper limits on the flux around the flashes $\left(F_{\text {pers }}\right)$. From these we infer the flash peak luminosities in terms of the Eddington luminosity $\left(L_{\text {Edd }}\right)$, assuming $L_{\mathrm{x}, \text { bol }} / L_{\text {Edd }} \equiv F_{\text {pers,bol }} / F_{\text {bb,peak }}($ see Sect. 3), where $F_{\text {pers,bol }}$ and $L_{\mathrm{x}, \text { bol }}$ are the bolometric pre-flash source flux and luminosity, respectively. Assuming $L_{\mathrm{Edd}}=2 \times 10^{38} \mathrm{erg} \mathrm{s}$ (see Sect. 2) we derive an upper limit on $L_{\mathrm{x}, \mathrm{bol}}$. In the last two columns we provide the time since the last and to the next detected X-ray outburst with respect to the time of the flash, $\Delta t_{\text {last }}$ and $\Delta t_{\text {next }}$, respectively. References are given in brackets: [1] this paper; [2] Conner et al. (1969); [3] Belian et al. (1972); [4] Cornelisse et al. (2002a); [5] Markwardt \& Swank (2004); [6] in 't Zand et al. (2003b); [7] Altamirano et al. (2008); [8] Markwardt et al. (2008); [9] Grebenev et al. (2005); [10] Markwardt \& Swank (2005); [11] Del Monte et al. (2008); [12] Cornelisse et al. (2007); [13] Jernigan (1976); [14] in 't Zand et al. (2001); [15] Marshall (1998).

sedimentation has the effect of increasing the $\dot{M}$ limits by about a factor of 2 (see Peng et al. 2007; Cooper \& Narayan 2007).

The minimum accretion rate below one expects no flashes at all (i.e., completely stable burning all the time), is even less well-studied nor well-determined, but it is probably of the order of $10^{-5} \dot{M}_{\text {Edd }}$ (see Fushiki \& Lamb 1987).

It is important to realize that the above quoted $\dot{M}$ values from flash theory are presumed to sustain for at least a few months (i.e., the thermal time scale of the crust). If there were a temporary increase in $\dot{M}$ on a shorter time scale, the temperature of the layer where the flash originates may still be higher than would be expected from the lower long-term averaged $\dot{M}$, thus influencing the flash behaviour.

\subsection{Comparing observations with theory}

We reiterate that, when comparing the observed limits on $\dot{M}$ with that inferred from flash theory, one has to keep in mind the uncertainties and assumptions mentioned at the end of Sect. 3.1, as well as the uncertainties in the flash theory. The minimum $\dot{M}$ below one expects no flashes, translates to an accretion luminosity of a few times $10^{33} \mathrm{erg} \mathrm{s}^{-1}$. This is interestingly close, but above, to that observed for our sample of sources, as well as other neutron star X-ray transients, in quiescence $\left(10^{31-33} \mathrm{erg} \mathrm{s}^{-1}\right.$; e.g., Cornelisse et al. 2002a,b, 2007; Campana et al. 2004; Jonker et al. 2007; Heinke et al. 2007, and references therein). The neutron stars in our source sample, therefore, must have been accreting at levels above that of quiescence, i.e., $\dot{M} \gtrsim 10^{-5} \dot{M}_{\text {Edd }}$. In this respect we note, however, the subluminous $\left(L_{\mathrm{bb}, \text { peak }} \sim\right.$ $4 \times 10^{36} \mathrm{erg} \mathrm{s}^{-1}$ ) flash seen from a source in M 28 with a pre-flash luminosity of $L_{\mathrm{x}, \text { bol }} \lesssim 10^{33} \mathrm{erg} \mathrm{s}^{-1}$ (Gotthelf \& Kulkarni 1997), which may counter the suggestion. For this source $L_{\mathrm{x}, \text { bol }} / L_{\mathrm{Edd}} \lesssim$ $5 \times 10^{-6}$ (assuming solar type compositions), which is about a factor of 1000 lower than the values found for the sources in our sample (Table 1).

The 1969 flash from Cen X-4 was bright, but we can not infer whether it had a photospheric radius-expansion phase (i.e., reached the Eddington limit). It could thus have been either a flash fueled by a deep layer of nearly pure He or a long mixed $\mathrm{H} / \mathrm{He}$ flash triggered by an unstable $\mathrm{H}$ flash. The expected $\dot{M}$ is thus $\lesssim 10^{-2} \dot{M}_{\text {Edd }}$, which is consistent with the observed upper limit based on the Vela $5 \mathrm{~B}$ non-detection prior to the flash (see also Hanawa \& Fujimoto 1986). This also holds for the flashes seen from 2S 1711-339, IGR J17473-2721 and 2S 1803-245, as well as those seen from the burst-only sources. The flashes from SAX J1808.4-3658 and GRS 1747-312 did reach the Eddington limit and lasted on the order of minutes (in 't Zand et al. 2001, 2003b). For these sources we thus infer that the expected $\dot{M}$ was between about a few times $10^{-3} \dot{M}_{\text {Edd }}$ and $10^{-2} \dot{M}_{\text {Edd }}$, roughly consistent with the observed upper limits on the pre-flash source luminosity. The expected $\dot{M}$ are 3-4 orders of magnitude higher than that inferred from observations in quiescence.

One can make simple analytic estimates how long such an enhanced accretion period has to last in order to show a flash (assuming the flash is produced from only the freshly accreted material, and that all of this fresh material is used during the flash). Burning $\mathrm{H}$ to $\mathrm{He}$ to $\mathrm{C}$ (using a $\mathrm{H}$ mass fraction $X=0.7$ and He mass fraction $Y=0.3$ ) gives roughly $5 \times 10^{18} \mathrm{erg} \mathrm{g}^{-1}$, whereas burning $\mathrm{He}$ to $\mathrm{C}$ (using $X=0, Y=1$ ) gives roughly $6 \times 10^{17} \mathrm{erg} \mathrm{g}^{-1}$ (see, e.g., Bildsten 1998). In the case of Cen X4's long 1969 flash, if it is due to unstable mixed H/He burning, we need at least $10^{21} \mathrm{~g}$ to get a flash fluence of more than $5 \times$ $10^{39} \mathrm{erg}$. If $\dot{M}$ onto the neutron star is about $10^{-4}-10^{-3} \dot{M}_{\mathrm{Edd}}$, one needs to wait at least 115-12 days for a flash to occur. If the flash is due to unstable pure He burning, one needs even more matter and one has to wait longer (assuming the steadily burning $\mathrm{H}$ does not leave He behind). Then the He mass needed is about $8 \times$ $10^{21} \mathrm{~g}$, and the neutron star needs to accrete at least an order of magnitude longer than estimated above. This enhanced accretion time scale grows inverse proportionally with mass accretion rate for even lower values of $\dot{M}$. Note that for $\dot{M} \lesssim 10^{-4} \dot{M}_{\mathrm{Edd}}$, in the mixed burning case, the time scale starts to become as long or even larger than the thermal time scale of the crust.

Inspection of Fig. 1 (right panel) shows, in the case of Cen X-4, no evidence of any increased X-ray activity (above about $10^{36} \mathrm{erg} \mathrm{s}^{-1}$ ), i.e., no enhanced accretion onto the neutron star, above the Vela $5 B$ detection level during the 40 days prior to the 1969 outburst (see also Conner et al. 1969). The other sources show comparable limits on the existence of temporary increase in $\dot{M}$, mainly based on observations with X-ray monitoring instruments such as the RXTE/ASM and BeppoSAX/WFC. However, these limits (more than 3 orders of magnitude higher than the quiescent luminosity) are not very constraining, and 
thus enhanced $\dot{M}$ could have occurred with maximum accretion luminosities of about $5 \times 10^{35}-10^{36} \mathrm{erg} \mathrm{s}^{-1}$, around the time of their flashes.

\subsection{Accretion during quiescence: conclusions}

We conclude that the presence of the flashes discussed here require an enhancement of the accretion rate above that of pure quiescence, either temporary or on longer time scales. In the first case the enhancement can occur in the phases just before or after the bright outburst, or be the result of a weak (presumably unobserved) outburst.

Indeed, Cornelisse et al. (2002a) already suggested that the burst-only sources either accrete persistently at "medium" $\dot{M}$ (with $L_{\mathrm{x}, \text { bol }} \simeq 10^{34-35} \mathrm{erg} \mathrm{s}^{-1}$ ), or that their flashes occur during or after faint outbursts (with $L_{\mathrm{x}, \mathrm{bol}} \lesssim 10^{36} \mathrm{erg} \mathrm{s}^{-1}$ ). Interestingly, one of the burst-only sources, XMMU J174716.1-281048, was in quiescence for some time and then started to accrete at low levels with $L_{\mathrm{x}, \mathrm{bol}} \simeq 6-10 \times 10^{34} \mathrm{erg} \mathrm{s}^{-1}$ (see Del Santo et al. 2007). Similarly, over the years AX J1754.2-2754 has been seen to accrete at low levels in between quiescence and outburst (Jonker \& Keek 2008, and references therein).

Contrary to the burst-only sources, the systems Cen X-4, IGR J17473-2721， 2S 1803-245， SAX J1808.4-3658, GRS 1747-312 and 2S 1711-339 have shown X-ray outbursts with $L_{\mathrm{x}, \text { bol }}>10^{36} \mathrm{erg} \mathrm{s}^{-1}$. Ten to eleven months after the SAX J1808.4-3658's 1998 outburst the system was in quiescence (e.g., Campana et al. 2002), but it had shown reflares after its 2000 and 2005 main outbursts (Wijnands et al. 2001; Campana et al. 2008). Also 2S 1711-339 has been detected at low-intensity levels at various times before and after its 1998/1999 X-ray outburst, with fluxes of about a factor of 25 below the peak outburst flux and about a factor of 3000 above quiescence (Cornelisse et al. 2002a; Wilson et al. 2003; Torres et al. 2004a,b). Another neutron star X-ray transient LMXB, 4U 1608-522, also shows low-intensity states (down by a factor of $\sim 10$ ) after its main outbursts, before going to quiescence (Keek et al. 2008). During its quiescence phase SAX J1808.4-3658 was several times measured to be about 10 times brighter than at other times (Campana et al. 2008). Quasi-persistent neutron star transients, such as KS 1731-260 and MXB 1659-29, have shown exponential decaying low-level intensity light curves after their main outburst over a period of several years (from about $2-5 \times 10^{33} \mathrm{erg} \mathrm{s}^{-1}$ to an apparent base level of $2-5 \times 10^{32} \mathrm{erg} \mathrm{s}^{-1}$; e.g., Cacket et al. 2006) ${ }^{8}$. All of the above suggests the possibility of ongoing accretion episodes after the main outburst, which may be universal among the (neutron-star) X-ray transients.

Although quiescence is reached some time after the X-ray outbursts of SAXJ1808.4-3658, GRS 1747-312 and 2 S 1711-339, we suggest that at the time of their flashes discussed in this paper $\dot{M}$ had not reached quiescent values yet. For 2S 1711-339 this was almost a year after the X-ray outburst (Table 1). On the other hand, it could be that at the end of the quiescent phase of Cen X-4, IGR J17473-2721 and 2S 1803-245, $\dot{M}$ was enhanced as predicted by the DIM (see Sect. 4$)$. No evidence is found of a long-term increase of the accretion rate; the increase is of temporary nature. It is difficult to estimate the

\footnotetext{
8 The smoothness of the decay of KS 1731-260 and MXB 1659-29 has been used by Cacket et al. (2006) as a counter argument for variations in $\dot{M}$ as the cause of it; they favour a cooling neutron star. We note, however, that the decay light curves are not too well sampled (effectively 5 observations over about 4 years).
}

duration of this temporary enhancement, however, due to insufficient time coverage and sensitivity.

\section{Do flashes trigger X-ray outbursts, or is it the opposite?}

Flashes have an impact on the region around the neutron star, i.e., the corona and/or the inner parts of the accretion disk. This can be seen in those flashes where the residual X-ray emission during the photospheric radius-expansion phase is lower than the pre-flash emission (e.g., Molkov et al. 2000; Strohmayer \& Brown 2002), and/or in those flashes where the decay shows pronounced irregularities from canonical exponential-like decay (e.g., van Paradijs et al. 1990; Strohmayer \& Brown 2002; Molkov et al. 2000; in 't Zand et al. 2005b, 2007; excluding those sources which are viewed at high inclination). We here pose the question whether a flash can be influential enough to enhance the accretion onto the neutron star; in other words, was the 1969 Cen X-4 flash the trigger of Cen X-4's subsequent X-ray outburst (and similarly for IGR J17473-2721 and 2S 1803-245)?

The answer depends on what causes outbursts in LMXBs. It is generally accepted that the physical mechanism driving these outbursts is analogous to that of dwarf novae (see Lasota 2001 for a review and references). The main difference is that in LMXBs irradiation by the central X-ray source strongly influences the disk's stability (van Paradijs 1996; Dubus et al. 1999) and the properties of the outburst cycle (Dubus et al. 2001).

According to the DIM, outbursts are driven by a thermalviscous instability which triggers heating fronts that bring an initially "cold" disk into a "hot", high accretion-rate, state. The hot outburst phase is ended by an inward-propagating cooling front which brings the disk back to a cold state. During the subsequent quiescent phase the disk fills up with matter until it reaches the instability limit, which corresponds to the critical accretion rate given by:

$\dot{M}_{\text {crit }}^{-}=2.64 \times 10^{15} \alpha_{0.1}^{0.01} R_{10}^{2.58} M_{1}^{-0.85} \mathrm{~g} \mathrm{~s}^{-1}$

where $\alpha=0.1 \alpha_{0.1}$ is the disk viscosity parameter, $R=$ $10^{10} \mathrm{~cm} R_{10}$ the radius and $M=1 M_{\odot} M_{1}$ the mass of the accreting object. The superscript "-" refers to the maximum accretion rate for stable cold disks (see Lasota et al. 2008).

During quiescence the accretion rate in the disk must everywhere be lower than the critical value given by Eq. (1). Therefore, for disks extending down to the neutron star surface (or to the innermost stable circular orbit) the resulting rates are ridiculously low, as mentioned in the Introduction. However, for disks truncated at, say, $\sim 10^{9} \mathrm{~cm}$, the accretion rate at the inner disk's edge is about $7 \times 10^{12} \mathrm{~g} \mathrm{~s}^{-1}$, i.e., $\sim 6 \times 10^{-6} \dot{M}_{\text {Edd }}$ (in agreement with observations of Cen X-4 in quiescence ${ }^{9}$ ). When the accretion rate reaches somewhere the critical value given by Eq. (1), an outburst starts. In LMXBs the outbursts always start near the inner edge of the (truncated) disk (see Dubus et al. 2001). In the model of Dubus et al. (2001) corresponding roughly to the parameters of Cen X-4, outbursts start at $\dot{M} \sim 2 \times 10^{14} \mathrm{~g} \mathrm{~s}^{-1}$ (see their Fig. 19), i.e., at $\dot{M} \sim 2 \times 10^{-4} \dot{M}_{\mathrm{Edd}}$, which, (consistently) implies an accumulation time for a critical pile of flash fuel of several months to more than a year, depending on the sort of flash produced (see Sect. 3.3).

\footnotetext{
${ }^{9}$ Given the $1.2 \mathrm{kpc}$ distance and a bolometric correction factor of 2 the observed X-ray luminosity in quiescence (see Sect. 2) corresponds to $L_{x, \text { bol }} \sim 3-10 \times 10^{32} \mathrm{erg} \mathrm{s}^{-1}$. This leads to inferred accretion rates in quiescence of about $2-5 \times 10^{12} \mathrm{~g} \mathrm{~s}^{-1}$, or $2-5 \times 10^{-6} \dot{M}_{\text {Edd }}$.
} 
In outburst (when accretion rates can reach Eddington limited values) the X-ray irradiation stabilizes the disk (van Paradijs 1996; Dubus et al. 1999), but in quiescence the X-ray accretion luminosity is too low to affect the stability properties. However, external disk irradiation (e.g., by a flash) can affect the disk's properties by significantly lowering the value of the critical density and accretion rate. In general, the irradiation temperature can be written as:

$\sigma T_{\mathrm{irr}}^{4}=C \frac{L_{x}}{4 \pi R^{2}}$,

where $L_{x}$ is the X-ray luminosity and $C$ represents the fraction of the $\mathrm{X}$-ray luminosity that heats up the disk and contains information on the irradiation geometry, the X-ray albedo and the X-ray spectrum. Based on observations of irradiated disks in low-mass X-ray binaries, Dubus et al. $(1999,2001)$ found that in outburst, when $L_{x}$ is the accretion luminosity, $C \approx 0.005$.

In general, however, the effect of varying X-ray luminosity can be mimicked by varying $C$. The upper continuous line in Fig. 15 of Dubus et al. (2001) shows the column-density profile of a disk just before the onset of the outburst: in its inner parts the density is very close to the critical one represented by a dotted line in that figure. This is the standard situation when irradiation in quiescence is negligible because of low accretion luminosity. The second (lower) dotted line in that figure corresponds to the case of a disk irradiated in quiescence ( $C$ multiplied by 100). In this case the outburst would have started at lower surfacedensity, i.e., earlier than in the case of a non-irradiated disk. Can this represent the effect of irradiation by a flash such as observed in Cen X-4?

As discussed in Sect. 3, the X-ray luminosity before the flash was not higher than $L_{x} \simeq 10^{36} \mathrm{erg} \mathrm{s}^{-1}$. Therefore, the effect of the flash can be roughly described by an increase in $C$ by a factor of at least 100 which corresponds to the situation represented at Fig. 15 of Dubus et al. (2001). The numerical values of the surface-density and truncation radius correspond to a $7 M_{\odot}$ accretor as it is the only available example in Dubus et al. (2001). The relative amplitude of the effect, however, does not depend on the mass of the compact object. So, in principle, a flash can accelerate the start of an outburst.

However, since the flash lasted only about $10 \mathrm{~min}$, one has to check that the disk had the time to modify its thermal structure, i.e., that it had enough time to react to irradiation. The characteristic thermal time scale of the disk is $t_{\mathrm{th}} \sim 1 /\left(\alpha \Omega_{\mathrm{K}}\right)$, where $\Omega_{\mathrm{K}}$ is the Keplerian angular velocity. Assuming an inner disk radius at $\sim 10^{9} \mathrm{~cm}$ and $\alpha=0.01$ one gets $t_{\text {th }} \sim 4 \mathrm{~min}$, which is just right, but a larger radius would preclude the flash significantly affecting the disk structure. ( $\alpha$ could have a value up to, say, 0.03 , but this would not help much).

As mentioned above, in the relevant non-irradiated model of Dubus et al. (2001), outbursts start at $\dot{M} \sim 2 \times 10^{14} \mathrm{~g} \mathrm{~s}^{-1}$. From Eq. (1) this corresponds to a radius of $\sim 4 \times 10^{9} \mathrm{~cm}$. Therefore, it is rather unlikely that the flash triggered the outburst. If anything, the opposite is true: according to the model, in the last two years preceding the outburst the accretion rate increases to a level, which may have provided the right conditions for the flash to occur (see above). One should, however, keep in mind that the exact value of the truncation radius depends on an "evaporation" mechanism model.

\section{Summary}

The detection of a flash at the end of the quiescent phases in Cen X-4, IGR J17473-2721 and 2S 1803-245, in between recurrent X-ray outbursts of SAX J1808.4-3658 and GRS 1747-312, and after an X-ray outburst of 2S 1711-339, shows that accretion was (temporarily) ongoing onto the neutron star. Such accretion must be at a level several orders of magnitudes higher than that inferred for quiescence, and the rates required are consistent with the truncated disk model which predicts an accretion-rate enhancement before the onset of the outburst. Therefore, it seems unlikely that crustal heating is the main source of luminosity in all of quiescence. Thus, care must be taken when considering the properties of a cooling neutron star when an outburst is over.

The DIM-predicted enhancement in accretion rate near the end of quiescence of about $10^{13}$ to $10^{14} \mathrm{~g} \mathrm{~s}^{-1}$ might have provided the right conditions for the flashes to occur in just before the outbursts of Cen X-4, IGR J17473-2721 and 2S 1803-245. The uncertainties inherent in the DIM do not allow us to test in detail the hypothesis that the flashes triggered or accelerated the start of, the outbursts (althought we regard it as unlikely), nor the 2-7-day delay between the flash and the X-ray outburst.

Acknowledgements. We thank Jorge Casares for discussions regarding the secondary star of Cen X-4, Randy Cooper regarding $\dot{M}$ limits from flash theory, Masaru Matsuoka regarding the flash seen from Cen X-4 by Hakucho, Paul Gorenstein and Rick Harnden for discussions about the Apollo 15 X-ray burst event, Arne Åsnes, Bob Lin, David Sibeck and Matt Taylor for discussions about solar wind particles and their interaction with the earth's magnetosphere, and Peter Jonker for commenting on an earlier draft of the paper. E.K. and J.Z. acknowledge support from the Faculty of the European Space Astronomy Centre (ESAC). J.P.L. was supported by the Centre National d'Études Spatiales (CNES).

\section{Appendix A: A flash from Cen X-4 in quiescence recorded by Apollo 15?}

\section{A.1. Apollo 15 observations}

The X-ray Fluorescence Spectrometer (1->3 keV), carried in the Scientific Instrument Module of the Command and Service Module on the Apollo 15 mission, was mainly used for orbital mapping of the lunar surface composition (see, e.g., Adler et al. 1972a,b, 1975). Galactic X-ray observations were made during the trans-Earth coast for several periods of about an hour (e.g., Adler et al. 1972b). Among the various other instruments were the Gamma-ray Spectrometer (500 keV-30 MeV; e.g., Arnold et al. 1972) and the Alpha Particle Spectrometer (4.5-9.0 MeV; e.g., Gorenstein \& Bjorkholm 1972). The X-ray Fluorescence Spectrometer was pointed towards various constellations over a period of about 3 days (1971, Aug. 5-7) with an attitude stability within a degree for approximately $1 \mathrm{~h}$ of observation (Adler et al. 1972b).

A large increase in the X-ray count rate for about 10 min was recorded on UT 1971, August 5, 00:34 (Gorenstein et al. 1974). The burst event occurred when the spacecraft was pointed towards the Centaurus constellation, for a period of about $65 \mathrm{~min}$ (Adler et al. 1972b; Gorenstein et al. 1974). According to the Apollo 15 Flight Journal $^{10}$, it was aimed at Centaurus A. The X-ray Fluorescence Spectrometer had all the known X-ray sources in Centaurus at that time in the full-width conal field of view of $50^{\circ 11}$ (Gorenstein et al. 1974); Cen X-4 was about $21.5^{\circ}$ from the center of the field of view.

At the peak of the burst event the intensity was several times higher than Sco X-1, and Gorenstein et al. (1974) noted that the

\footnotetext{
10 See http://history.nasa.gov/ap15fj/

11 The nominal field of view is about $60^{\circ}$ full-width at half-maximum (see, e.g., Adler et al. 1972a,b); in practice, however, it proved out to be more complicated (Adler et al. 1975).
} 


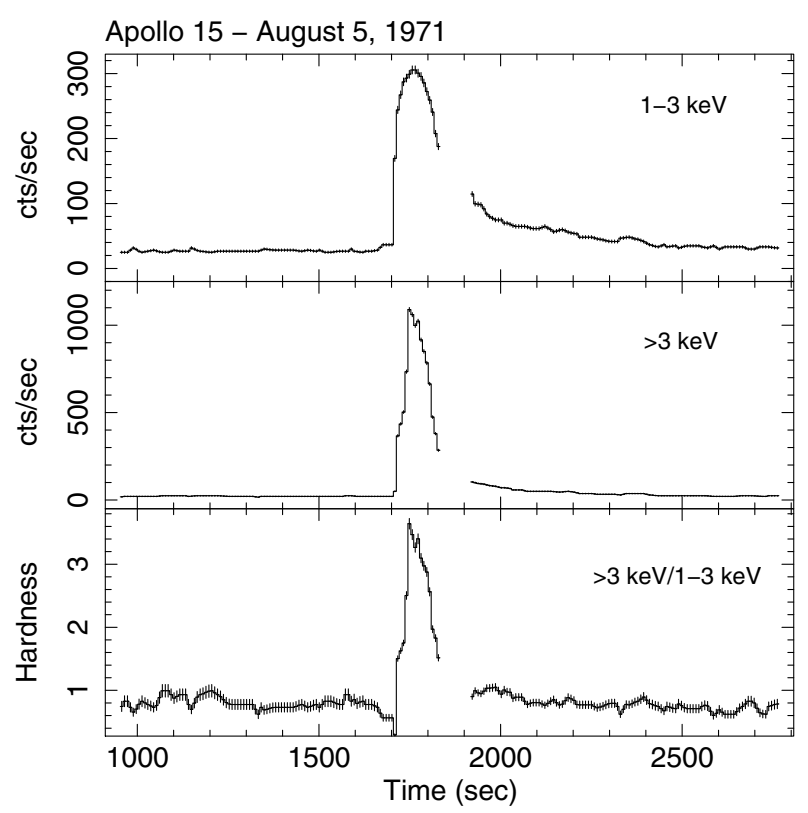

Fig. A.1. Top and middle: light curves recorded by the X-ray Fluorescence Spectrometer on-board Apollo 15 in the $1-3 \mathrm{keV}$ band and at $>3 \mathrm{keV}$ (taken from Gorenstein et al. 1974). Bottom: hardness curve derived from the ratio of the count rates at $>3 \mathrm{keV}$ and in the $1-3 \mathrm{keV}$ band. Data were recorded every $8 \mathrm{~s}$. The gap in the curves are due to observations of the on-board calibration source. $t=0 \mathrm{~s}$ corresponds to UT 1971, Aug. 5, 00:04.

light curve and strength of the burst event was similar to that reported for the flash from Cen X-4 by Belian et al. (1972). Based on the above characteristics Gorenstein et al. (1974) suggested their event to be a similar flash from Cen X-4.

The burst event was observed in the $1-3 \mathrm{keV}$ band, at $>3 \mathrm{keV}$, and at $>7 \mathrm{keV}$. Unfortunately, there were no soft particle detectors on-board to discriminate between local and Galactic radiation, so a passage through a region of particles in cis-lunar space could not be ruled out. However, the background level before and after the event was rather stable (Gorenstein et al. 1974). No additional events were seen by the Gamma-ray Spectrometer and the Alpha Particle Spectrometer, suggesting the event was of X-ray origin, supported by the sparse energy information of the X-ray Fluorescence Spectrometer. In Sect. A.2 we elaborate further on this issue.

We digitized the data shown in Fig. 1 by Gorenstein et al. (1974), which gives the events in the $1-3 \mathrm{keV}$ band and at $>3 \mathrm{keV}$ (see Fig. A.1, top panels). We assumed the uncertainties to be due to pure Poisson statistics, but note that the systematic uncertainties are larger due to the pointing inaccuracy and non-uniform collimator response. We derived the hardness curve by taking the ratio of the count rates at $>3 \mathrm{keV}$ over the count rates in the 1-3 keV band (see Fig. A.1, bottom panel). Clearly, the event shows a fast-rise $(\simeq 50$ s from start to peak) and exponential-like decay (decay time of $\simeq 100 \mathrm{~s}$ and $\simeq 25 \mathrm{~s}$, respectively in the $1-3 \mathrm{keV}$ band and at $>3 \mathrm{keV}$, from about $t=1800 \mathrm{~s}$ to $2000 \mathrm{~s}$ ). During the rise to peak the source first hardens; subsequently, during the decay the source softened. Indeed, this is typically seen for thermonuclear flashes (see Sect. 2).

The event's peak rate is about 2450 and 8500 cts per $8 \mathrm{~s}$ in the 1-3 keV band and at $>3 \mathrm{keV}$, respectively, while Sco X-1 varied between 1350-2050 and 2000-2750 cts per 8 s, respectively, as seen later on Aug. 5 (see Adler et al. 1972b). This correspond to a total peak rate about 2.3-3.3 times that of Sco X-1. The above quoted rates are uncorrected for collimator response, therefore, if the event came from Cen X-4 the actual peak may be up to about a factor of 2 or so higher. Measurements by the RXTE AllSky Monitor (ASM) show that the daily average flux of Sco X-1 is roughly $11-13 \mathrm{Crab}(2-12 \mathrm{keV})$. The Apollo 15 burst event peak flux is thus indeed in the range observed for the flashes seen by Vela $5 B$ (Belian et al. 1972) and Hakucho (Matsuoka et al. 1980). Although there were a couple of (currently) known flash sources (see, e.g., in 't Zand et al. 2004) in the field of view of the X-ray Fluorescence Spectrometer at the time of the event, they are all known to not show flashes like that seen from Cen X4 , and certainly not as bright.

Cen X-4 was in quiescence around the time of the event (see Sect. 2). During the event itself Vela $5 B$ was operating, but did not look at Cen X-4's region. Prompted by the Apollo 15 findings, we searched the Vela $5 B$ data base for similar flashes as seen in 1969 (see Sect. 2), but found none. Also the RXTE/ASM data base on Cen X-4 does not reveal any similar events above the level of about $0.5 \mathrm{Crab}$. It would be interesting to find out if similar events exist in other databases, such as of CGRO/BATSE.

\section{A.2. Particle event or not?}

Energetic electron bursts at energies of keV to tens, or even hundredths, of $\mathrm{keV}$ (but below about $500 \mathrm{keV}$ ) are common around the Earth's magnetosphere. They occur especially in the Earth's magnetotail in the anti-Sun direction, but also in the magnetosheath which extends to $\pm \sim 30^{\circ}$ from the anti-Sun direction, and around the bow shock (to $\pm \sim 45^{\circ}$ from anti-Sun); see, e.g., Sarris et al. (1976), Baker \& Stone (1978), Meng et al. (1981). They arise during geomagnetic activity. If Apollo 15 was in these regions then there is a likelihood of such a burst; either the spacecraft passed through a static particle region or such a region swept across the spacecraft.

The position of the Sun and Moon on Aug. 5, 1971, i.e., the longitude $\phi$ and latitude $\lambda$ in ecliptic coordinates were roughly $\phi, \lambda=132^{\circ}, 0^{\circ}$ and $\phi, \lambda=288^{\circ},-2^{\circ}$, respectively. The EarthMoon distance was around $374307 \mathrm{~km}$ on that day. According to the Apollo 15 Flight Journal $^{10}$ the spacecraft was about $15200 \mathrm{~km}$ from the Moon around the time of the Centaurus region observations, so we may assume it was close to the position of the Moon. Apollo 15 was thus about $24^{\circ}$ from the anti-Sun direction, placing it near the boundaries of the Earth's magnetotail.

The Interplanetary magnetic field was southward on Aug. 5, $1971^{12}$, favouring geomagnetic activity and the production of energetic particles in the Earth's magnetosphere. This was particularly true for the first half day, from UT 00:00-12:00. However, the activity was not exceptional in any way, peaking from UT 03:00-04:00 and gradually decaying the rest of the day. Further inspection ${ }^{13}$ also reveals a moderately strong geomagnetic substorm in progress at this time and a weak geomagnetic storm.

If the burst event originated from increased geomagnetic activity, one would expect, however, to see more such events. Gorenstein et al. (1974) report that, apart from the 1971, Aug. 5 event, during a total about $20 \mathrm{~h}$ of observing time of the X-ray Fluorescence Spectrometers onboard Apollo 15 and Apollo 16, two other events of two minute durations were seen, as well as one other event of longer duration. Also, as mentioned in Sect. B.1, the X-ray flux was reported to be stable before and

\footnotetext{
12 See http://omniweb.gsfc.nasa.gov

13 See

http://swdcwww.kugi .kyoto-u.ac.jp/dstae/index.html
} 
after the event. This suggests that the Aug 5 burst event is a rather isolated event. Moreover, a changing slope of the spectrum of a particle burst in the magnetotail is not likely to produce a dramatic change of spectral hardness, as observed in this burst event. We, therefore, conclude that the Aug. 5 burst event has most probably a celestial origin.

\section{References}

Adler, I., Trombka, J., Gerard, J., et al. 1972a, in Apollo 15: Preliminary Science Report, NASA SP-289, 17

Adler, I., Gerard, J., Trombka, J., et al. 1972b, in Proceedings of the Third Lunar Science Conference, 3, 2157

Adler, I., Schmadebeck, R., Trombka, J. I., Gorenstein, P., \& Bjorkholm, P. 1975, Space Sci. Instrum., 1, 305

Altamirano, D., Degenaar, N., in 't Zand, J., Markwardt, C., \& Wijnands, R. 2008, ATel \#1459

Arnold, J. R., Peterson, L. E., Metzger, A. E., Trombka, J.I. 1972, in Apollo 15: Preliminary Science Report, NASA SP-289, 16

Baker, D. N., \& Stone, E. C. 1978, JGR, 83, 4327

Bildsten, L. 1998, in The Many Faces of Neutron Stars, ed. R. Buccheri, J. van Paradijs, \& M. A. Alpar (Kluwer Academic Publishers), 419

Bildsten, L., \& Chakrabarty, D. 2001, ApJ, 557, 292

Belian, R. D., Conner, J. P., \& Evans, W. D. 1972, 171, L87

Belian, R. D., Conner, J. P., \& Evans, W. D. 1975, 206, L135

Brown, E. F., Bildsten, L., \& Rutledge, R. E. 1998, ApJ, 504, L95

Cacket, E. M., Wijnands, R., Linares, M., et al. 2006, MNRAS, 372, 479

Canizares, C. R., McClintock, J. E., \& Grindlay, J. E. 1980, ApJ, 236, L55

Campana, S., Mereghetti, S., Stella, L., \& Colpi M. 1997, A\&A, 324, 941

Campana, S., Stella, L., Gastaldello, F., et al. 2002, ApJ, 575, L15

Campana, S., Israel, G. L., Stella, L., Gastaldello, F., \& Mereghetti, S. 2004 ApJ, 601, 474

Campana, S., Stella, L., \& Kennea, J. A. 2008, ApJ, 684 L99

Casares, J., Bonifacio, P., González Hernández, J. I., Molaro, P., \& Zoccali, M. 2007, A\&A, 470, 1033

Chenevez, J., Falanga, M., Brandt, S., et al. 2006, A\&A, 449, L5

Chevalier, C., \& Ilovaisky, S. 1990, A\&A, 228, 115

Chevalier, C., Ilovaisky, S. A., van Paradijs, J., Pedersen, H., \& van der Klis, M. 1989, A\&A, 210, 114

Cocchi, M., Bazzano, A., Natalucci, L., et al. 2001, A\&A, 378, L37

Conner, J. P., Evans, W. D., \& Belian, R. D. 1969, ApJ, 157, L157

Cooper, R. L., \& Narayan, R. 2007, ApJ, 661, 468

Cornelisse, R., Verbunt, F., in 't Zand, J. J. M., et al., 2002a, A\&A, 392, 885

Cornelisse, R., Verbunt, F., in 't Zand, J. J. M., Kuulkers, E., \& Heise, J. 2002b, A\&A, 392, 931

Cornelisse, R., in 't Zand, J. J. M., Verbunt, F., et al. 2003, A\&A, 405, 1033

Cornelisse, R., Wijnands, R., \& Homan, J. 2007, MNRAS, 380, 1637

Cumming, A. 2003, ApJ, 595, 1077

Czerny, M., Czerny, B., \& Grindlay, J. 1987, ApJ, 312, 122

Del Monte, E., Evangelista, Y., Feroci, M., et al. 2008, ATel \#1445

Del Santo, M., Sidoli, L., Mereghetti, S., Bazzano, A., Tarana, A., Ubertini, P. 2007, A\&A, 468, L17

Done, C. 2002, Phil. Trans. R. Soc. Lond. A, 360, 1967

Dubus, G., Lasota, J.-P., Hameury, J.-M., \& Charles, P. A. 1999, MNRAS, 303, 139

Dubus, G., Hameury, J.-M., \& Lasota, J.-P. 2001, A\&A, 373, 251

Evans, W. D., Belian, R. D., \& Conner, J. P. 1970, ApJ, 159, L57

Fabbiano, G., \& Branduardi, G. 1979, ApJ, 227, 294

Falanga, M., Chenevez, J., Cumming, A., Kuulkers, E., Trap, G., Goldwurm, A. 2008, A\&A, 484, 43

Fisker, J. L., Schatz, H., \& Thielemann, F.-K. 2008, ApJS, 174, 261

Forman, W., Jones, C., Cominsky, L., et al. 1978, ApJS, 38, 357

Frank, J., King, A., \& Raine, D. 1992, in Accretion Power in Astrophysics, 2nd edn., Cambridge Astrophys. Ser. 21, CUP

Fujimoto, M. Y., Hanawa, T., \& Miyaji, S. 1981, ApJ, 247, 267

Fushiki, I., \& Lamb, D. Q. 1987, ApJ, 323, L55

Galloway, D. K., Muno, M. P., Hartman, J. M., Psaltis, D., \& Chakrabarty, D. 2008, ApJS, 179, 360

González Hernández, J. I., Rebolo, R., Peñarrubia, J., Casares, J., \& Israelian, G. 2005a, A\&A, 435, 1185

González Hernández, J. I., Rebolo, R., Israelian, G., et al. 2005b, ApJ, 630, 495
Gorenstein, P., \& Bjorkholm, P. 1972, in Apollo 15: Preliminary Science Report, NASA SP-289, 18

Gorenstein, P., Bjorkholm, P., \& Harnden, F. R. Jr. 1974, in Proceedings of the Conference on Transient Cosmic Gamma- and X-ray Sources, ed. I. B. Strong, Los Alamos Scientific Laboratory, University of California, Los Alamos, New Mexico, LA-5505-C Conf. Proc., UC-34b, 74

Gotthelf, E. V., \& Kulkarni, S. R. 1997, ApJ, 490, L161

Grebenev, S. A., Molkov, S. V., \& Sunyaev, R. A. 2005, ATel \#467

Grindlay, J., Gursky, H., Schnopper, H., et al. 1975, ApJ, 205, L127

Hanawa, T., \& Fujimoto, M. Y. 1986, PASJ, 38, 13

Heger, A., Cumming, A., Galloway, D. K., \& Woosley, S. E. 2007, ApJ, 671, L141

Heinke, C. O., Jonker, P. G., Wijnands, R., \& Taam, R. E. 2007, ApJ, 660, 1424

Hoffman, J. A., Marshall, H. L., \& Lewin, W. H. G. 1978, Nature, 271, 630

Hjellming, R. M. 1979, IAUC \# 3369

in 't Zand, J. J. M., Cornelisse, R., Kuulkers, E., et al. 2001, A\&A, 372, 916

in 't Zand, J. J. M., Hulleman, F., Markwardt, C. B., et al. 2003a, A\&A, 406, 233

in 't Zand, J. J. M., Strohmayer, T. E., Markwardt, C. B., \& Swank, J. 2003b, A\&A, 409, 659

in 't Zand, J., Verbunt, F., Heise, J., et al. 2004, Nuc. Phys. B (Proc. Suppl.), 132, 486

in 't Zand, J. J. M., Cumming, A., van der Sluys, M. V., Verbunt, F., \& Pols, O. R. 2005, A\&A, 441, 675

in 't Zand, J. J. M., Jonker, P. G., \& Markwardt, C. B. 2007, 465, 953

Jernigan, G. 1976, IAU Circ. 2957

Jonker, P. G. 2008, in 40 Years of Pulsars: Millisecond Pulsars, Magnetars and More, AIP Conf. Proc. 983, 519

Jonker, P. G., \& Keek, L. 2008, ATel \#1643

Jonker, P. G., Steeghs, D., Chakrabarty, D., \& Juett, A. M. 2007, ApJ, 665, L147 Joss, P. C. 1977, Nature, 270, 310

Kaluzienski, L. J., Holt, S. S., \& Swank, J. H. 1980, ApJ, 241, 779

Keek, L., in 't Zand, J. J. M., Kuulkers, E., et al. 2008, A\&A, 479, 177

Kirsch, M. G., Briel, U. G., Burrows, D., et al. 2005, in UV, X-ray and Gammaray Space Instrumentation for Astronomy XIV, ed. O. H. W. Siegmund, Proc. SPIE, 5898, 22

Kuulkers, E. 2004, Nuc. Phys. B Proc. Suppl., 132, 466

Lamb, D. Q., \& Lamb, F. K. 1978, ApJ, 220, 291

Lasota, J.-P. 2001, NewAR, 45, 449

Lasota, J.-P., Narayan, R., \& Yi, I. 1996, A\&A, 314, 813

Lasota, J.-P., Dubus, G., \& Kruk, K. 2008, A\&A, 486, 523

Lewin, W. H. G., van Paradijs, J., \& Taam, R. E. 1993, SSRv, 62, 223

Liu, B. F., Mineshige, S., Meyer, F., Meyer-Hofmeister, E., \& Kawaguchi, T. 2002, ApJ, 575, 117

Markwardt, C. B., \& Swank, J. H. 2005, ATel \#498

Markwardt, C. B., Altamirano, D., Swank, J. H., \& in 't Zand, J. 2008, ATel \#1460

Maraschi, L., \& Cavaliere, A. 1977, Highlights of Astronomy, 4, 127

Marshall, F. E. 1998, IAU Circ. 6876

Matsuoka, M., Inoue, H., Koyama, K., et al. 1980, ApJ, 240, L137

Meng, C.-I., Lui, A. T. Y., Krimigis, S. M., Ismail , S., \& Williams, D. J. 1981, JGR, 86, 5682

Menou, K., Esin, A. A., Narayan, R., et al. 1999, ApJ, 520, 276

Molkov, S. V., Grebenev, S. A., \& Lutovinov, A. A. 2000, A\&A, 357, L41

Molkov, S., Revnivtsev, M., Lutovinov, A., \& Sunyaev, R. 2005, A\&A, 434, 1069

Narayan, R., \& McClintock, J. E. 2008, NewAR, 51, 733

Osaki, Y. 1974, PASJ, 26, 429

Peng, F., Brown, E. F., \& Truran, J. W. 2007, ApJ, 654, 1022

Priedhorsky, W. C., \& Holt, S. S. 1987, SSRv, 45, 291

Rutledge, R. E., Bildsten, L., Brown, E. F., Pavlov, G. G., \& Zavlin, V. E. 2001, ApJ, 551, 921

Sarris, E. T., Krimigis, S. M., \& Armstrong, T. P. 1976, JGR, 81, 2341

Strohmayer, T., \& Bildsten, L. 2006, in Compact stellar X-ray sources, ed.

W. H. G. Lewin, \& M. van der Klis, Cambridge Astrophys. Ser. 39, 113

Strohmayer, T. E., \& Brown, E. F. 2002, ApJ, 566, 1045

Torres, M. A. P., McClintock, J. E., Garcia, M. R., et al. 2004a, ATel \#233

Torres, M. A. P., McClintock, J. E., Garcia, M. R., et al. 2004b, ATel \#238 van Paradijs, J. 1996, ApJ, 464, L139

van Paradijs, J., Dotani, T., Tanaka, Y., \& Tsuru, T. 1990, PASJ, 42, 633

van Paradijs, J., Verbunt, F., Shafer, R. A., \& Arnaud, K. A. 1987, A\&A, 182, 47

Whitlock, L. A., \& Tyler, P. 1994, Legacy, 4, 31

Whitlock, L., Lochner, J., \& Rhode, K. 1992, Legacy, 2, 25

Wijnands, R., Méndez, M., Markwardt, C., et al. 2001, ApJ, 560, 892

Wilson, C. A., Patel, S. K., Kouveliotou, C., et al. 2003, ApJ, 596, 1220

Woosley, S. E., Heger, A., Cumming, A., et al. 2004, ApJS, 151, 75 